\title{
Multidisciplinary Applications of Detached-Eddy Simulation to Separated Flows at High Reynolds Numbers
}

\author{
Scott A. Morton, Matt Steenman, \\ and Russell M. Cummings \\ USAF Academy, Colorado \\ Springs, $C O$ \\ $\{$ Scott.Morton, \\ Matthew.Steenman\}@usafa.af.mil, \\ Russell.Cummings@usafa.edu \\ James R. Forsythe and Kenneth \\ E. Wurtzler \\ Cobalt Solutions, $L L C$, \\ Springfield, $\mathrm{OH}$ \\ \{forsythe, \\ wurtzler\}@cobaltcfd.com
}

\author{
Kyle D. Squires \\ Arizona State University, \\ Tempe, $A Z$ \\ squires@asu.edu
}

\author{
Shawn H. Woodson \\ Naval Air Systems Command, Patuxent River, MD \\ woodsonsh@navair.navy.mil
}

\author{
Philippe R. Spalart \\ Boeing Commercial Airplanes, Seattle, WA \\ Philippe.r.Spalart@boeing.com
}

\begin{abstract}
This work focuses on multidisciplinary applications of Detached-Eddy Simulation (DES), principally flight mechanics and aeroelasticity. Specifically, the lateral instability (known as abrupt wing stall) of the preproduction F/A-18E is reproduced using DES, including the unsteady shock motion. The presence of low frequency pressure oscillations due to shock motion in the current simulations and the experiments motivated a full aircraft calculation, which showed low frequency high-magnitude rolling moments that could be a significant contributor to the abrupt wing stall phenomenon. DES is also applied to the F-18 high angle of attack research vehicle (HARV) at a moderate angle of attack to reproduce the vortex breakdown leading to vertical stabilizer buffet. Unsteady tail loads are compared to flight test data. This work lays the foundation for future deforming grid calculations to reproduce the aero-elastic tail buffet seen in flight test. Solution based grid adaption is used on unstructured grids in both cases to improve the resolution in the separated region.

Previous DoD Challenge work has demonstrated the unique ability of the DES turbulence treatment to accurately and efficiently predict flows with massive separation at flight Reynolds numbers. DES calculations have been performed using the Cobalt code and on unstructured grids, an approach that can deal with complete configurations with very few compromises. A broad range of flows has been examined in previous Challenge work, including aircraft forebodies, airfoil sections, a missile afterbody, vortex breakdown on a delta
\end{abstract}

wing, and the F-16 and F-15E at high angles-of-attack. All DES predictions exhibited a moderate to significant improvement over results obtained using traditional Reynolds-averaged models and often excellent agreement with experimental/flight-test data. DES combines the efficiency of a Reynolds-averaged turbulence model near the wall with the fidelity of Large-Eddy Simulation (LES) in separated regions. Since it uses Large-Eddy Simulation in the separated regions, it is capable of predicting the unsteady motions associated with separated flows. The development and demonstration of improved methods for the prediction of flight mechanics and aeroelasticity in this Challenge is expected to reduce the acquisition cost of future military aircraft.

\section{Introduction}

Numerical simulations are an important tool for predicting aircraft performance, especially in off-design regimes that are difficult to investigate using wind-tunnel or flight testing. While CFD for aerodynamic applications is coming of age at various labs and in universities, e.g., full-airplane computations are now possible, one of the main stumbling blocks to the increased use of CFD for design and analysis has been an inability to accurately predict the unsteady effects of massive flow separations. Recent efforts on predicting massively separated flows around full aircraft at flight Reynolds numbers, however, has shown that Detached-Eddy Simulation (DES) is a viable method for use in this difficult flow regime. The present investigators have predicted the massively 
separated flow over several aircraft with DES predictions in good agreement with experiments or flight-test data (Forsythe et $a l^{[1]}$, Squires et $a l .{ }^{[2]}$ ). These successful efforts motivate the present research - extension of DES to multidisciplinary applications. The two applications considered are flight mechanics and aeroelasticity. The algorithm requirements in extending the current simulation methodology into these areas are similarprincipally the use of grid speed terms. The present work focuses on laying the foundation for subsequent grid motion (both deforming and rigid body) calculations. The cases considered are the abrupt wing stall (AWS) of the pre-production F/A-18E and vortex breakdown of the F18C. This represents both flight mechanics (lateral instability) and aeroelasticity (tail buffet) of full aircraft.

\section{Problem and Methodology}

\subsection{Abrupt Wing Stall.}

During envelope expansion flights of the F/A-18E/F in the Engineering and Manufacturing Development phase, the aircraft encountered uncommanded lateral activity, which was labeled "wing drop". An extensive resolution process was undertaken by the Navy and its contractors to resolve this issue. A production solution was developed, which included revising the flight control laws and the incorporation of a porous wing fold fairing to eliminate the wing drop tendencies of the preproduction F/A-18E/F. The wing drop events were traced to an abrupt wing stall (AWS) on either the left or right wing panel, causing a sudden and severe roll-off in the direction of the stalled wing. An important distinction between wing drop and AWS is that wing drop is the dynamic response of an aircraft to an aerodynamic event, while AWS is an aerodynamic event that can trigger a wing drop. ${ }^{[3]}$

Unsteady measurements on a model of a preproduction F/A-18E were made by Schuster and Byrd $^{[4]}$, motivated by the following statement: "Since AWS and the resulting lateral instabilities are dynamic or, at best highly sensitive quasi-static phenomena, measurement of unsteady wing surface pressures, loads, and accelerations were incorporated into the test procedures to investigate the potential unsteady causes and/or indicators of AWS." The initial findings from these tests showed highly unsteady surface pressures indicative of shock oscillation.

Unsteady shock oscillations have been highlighted by Dolling $^{[5]}$ as a problem for steady state methods. The supersonic separated compression ramp pulses at low frequency. The resulting time-averaged surface pressures are smeared due the time averaging of a moving shock. Accurately predicting this flow has eluded CFD researchers for decades. Dolling ${ }^{[5]}$ suggests that better agreement with time-averaged experimental data could be obtained if the CFD simulation included the global unsteadiness of the shock motion, then took a time average. This is the approach that is taken in the current research.

Besides obtaining an improved time-averaged prediction, however, it is also desired to complement unsteady wind tunnel methods ${ }^{[4]}$ with CFD to gain further insight into the potential of the unsteady flow to contribute to the AWS phenomena. The CFD complements the experiments by providing results unaffected by aeroelastic effects, and more detailed flow visualizations. The baseline case considered is an $8 \%$ model of a preproduction F/A-18E with $10^{\circ} / 10^{\circ} / 5^{\circ}$ flaps (leading-edge flaps/trailing-edge flaps/aileron flaps) at Mach 0.9 and no tails. DES calculations are performed on a baseline and adapted grid and compared to unsteady wind tunnel measurements and RANS models. Although not a comprehensive validation, confidence is built in the DES method for this class of flow.

In order to obtain approval for releasing this paper to the public, quantitative information has been removed from most vertical scales.

\subsection{Vortex Breakdown.}

The F-18 High Angle of Attack Research Vehicle (HARV; see Figure 1) has proven to be an excellent source of data for researchers working on high angle of attack flowfields. ${ }^{[6,7,8]}$ Extensive flight testing of the HARV has been conducted that provides a rich source of flow visualization, surface pressures, and aeroelastic information. The F-18 utilizes wing leading edge extensions (LEX) to generate vortices which enhance the wing lift, and the twin vertical tails are canted to intercept the strong vortex field and increase maneuverability. At large incidence, the LEX vortices breakdown upstream of the vertical tails, resulting in a loss of yaw control power and severe aeroelastic effects. ${ }^{[9]}$ This tail buffet phenomenon was reduced by using extensive flight tests to design a LEX fence. The ultimate goal of computationally modeling the flowfield shown in Figure 1 would be to accurately simulate the aeroelastic impact of the LEX vortices on the twin vertical tails. The current level of simulation technology, however, has not allowed for accurate prediction of vortex breakdown, and the unsteady flow downstream of breakdown, at flight Reynolds numbers. 


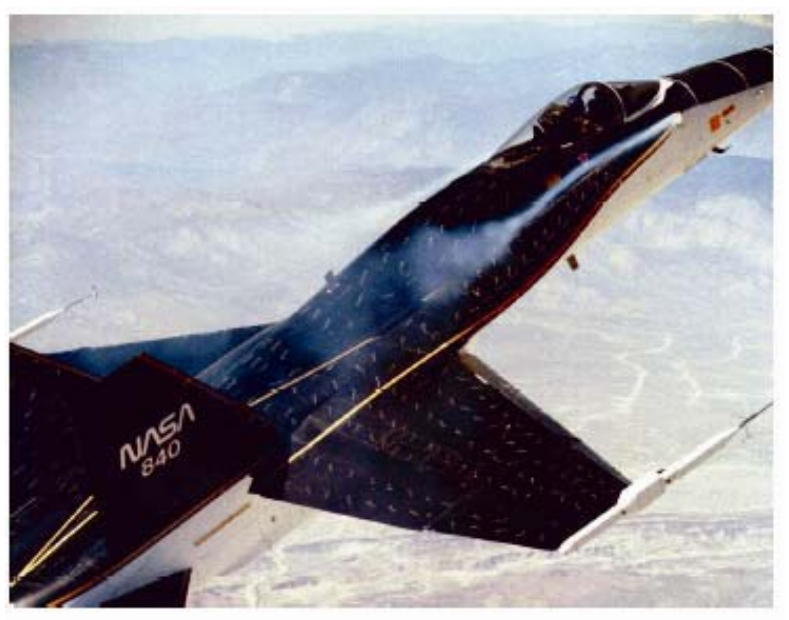

Figure 1. NASA F-18 High Angle of Attack Research Vehicle (HARV).

The specific aim of this work is to test the accuracy and efficiency of DES in predicting vortex breakdown over a full aircraft. This works builds on previous successful work on vortex breakdown over a delta wing $^{[26]}$. Another goal of the work is to apply adaptive mesh refinement (AMR) to this challenging flow. Computations are made for the F-18C at $\alpha=30^{\circ}, M_{\infty}=$ 0.2755 , and $R e_{\infty}=13.9 \times 10^{6}$ which determine the importance of highly refined grids (including automatic mesh refinement) on the accurate prediction of complex vortical flowfields. Comparisons are made between steady Reynolds-averaged Navier-Stokes (RANS), unsteady Reynolds-averaged Navier-Stokes (U-RANS), and Spalart-Allmaras DES (SADES), and the resulting predictions are compared with available flight test data for the F-18 HARV.

\subsection{Flow Solver.}

The commercial unstructured flow solver Cobalt was chosen because of its speed and accuracy. Strang et al. $\left.{ }^{[11}\right]$ validated the numerical method on a number of problems, including the Spalart-Allmaras model (which forms the core of the DES model). Tomaro et al. ${ }^{[12]}$ converted the code from explicit to implicit, enabling CFL numbers as high as one million. Grismer et al. ${ }^{[13]}$ then parallelized the code, yielding a linear speedup on as many as 1024 processors. Forsythe et al. ${ }^{[14]}$ provided a comprehensive testing and validation of the RANS models: SpalartAllmaras, Wilcox's $k-\omega$, and Menter's models. The Parallel METIS (ParMetis) domain decomposition library of Karypis and Kumar ${ }^{[15]}$ and Karypis et al. ${ }^{[16]}$ is also incorporated into Cobalt. ParMetis divides the grid into nearly equally sized zones that are then distributed among the processors.
The numerical method is a cell-centered finitevolume approach applicable to arbitrary cell topologies (e.g., hexahedrals, prisms, tetrahdra). The spatial operator uses the exact Riemann Solver of Gottlieb and Groth ${ }^{[17]}$, least squares gradient calculations using QR factorization to provide second-order accuracy in space, and TVD flux limiters to limit extremes at cell faces. A point implicit method using analytic first-order inviscid and viscous Jacobians is used for advancement of the discretized system. For time-accurate computations, a Newton subiteration scheme is employed, and the method is secondorder accurate in time.

The compressible Navier-Stokes equations were solved in an inertial reference frame. To model the effects of turbulence, a turbulent viscosity $\left(\mu_{t}\right)$ is provided by the turbulence model. To obtain $k_{t}$ (the turbulent thermal conductivity), a turbulent Prandtl number is assumed with the following relation: $\operatorname{Pr}_{t}=\frac{c_{p} \mu_{t}}{k_{t}}=0.9$. In the governing equations, $\mu$ is replaced by $\left(\mu+\mu_{t}\right)$ and $k$ (the thermal conductivity) is replaced by $\left(k+k_{t}\right)$. The laminar viscosity, $\mu$, is defined using Sutherland's law.

\subsection{Reynolds-Averaged Models.}

In order to provide a baseline for comparison, computations were performed with two of the leading Reynolds-averaged models. The first model used was the Spalart- Allmaras (SA) one-equation model ${ }^{[18]}$. This model solves a single partial differential equation for a variable $\widetilde{v}$ which is related to the turbulent viscosity. The differential equation is derived by, "using empiricism and arguments of dimensional analysis, Galilean invariance and selected dependence on the molecular viscosity." The model includes a wall destruction term that reduces the turbulent viscosity in the log layer and laminar sublayer, and trip terms that provide a smooth transition from laminar to turbulent. For the current research, the trip term was turned off, and the flow assumed fully turbulent.

The second model used was Menter's Shear Stress Transport (SST) model ${ }^{[19,20]}$. The method is a blend of a $k-\epsilon$ and $k-\omega$ model which uses the best features of each model. The model uses a parameter $F_{1}$ to switch from $k-$ $\omega$ to $k-\epsilon$ in the wake region to prevent the model from being sensitive to freestream conditions. The implementation used includes a compressibility correction as detailed in Forsythe et al. ${ }^{[14]}$.

\subsection{Detached-Eddy Simulation.}

The original DES formulation is based on a modification to the Spalart-Allmaras RANS model ${ }^{[18]}$ such that the model reduces to its RANS formulation near 
solid surfaces and to a subgrid model away from the wall ${ }^{[21]}$. The basis is to attempt to take advantage of the usually adequate performance of RANS models in the thin shear layers where these models are calibrated and the power of LES for resolution of geometry-dependent and three-dimensional eddies. The DES formulation is obtained by replacing in the S-A model the distance to the nearest wall, $d$, by $\widetilde{d}$ where $\tilde{d}$ is defined as

$$
\widetilde{d} \equiv \min \left(d, C_{D E S} \Delta\right)
$$

In Eqn. (1), for the computations performed in this project, $\Delta$ is the largest distance between the cell center under consideration and the cell center of the neighbors (i.e., those cells sharing a face with the cell in question). In "natural" applications of DES, the wall-parallel grid spacings (e.g., streamwise and spanwise) are at least on the order of the boundary layer thickness and the S-A RANS model is retained throughout the boundary layer, i.e., $\widetilde{d}=d$. Consequently, prediction of boundary layer separation is determined in the 'RANS mode' of DES. Away from solid boundaries, the closure is a one-equation model for the sub-grid-scale (SGS) eddy viscosity. When the production and destruction terms of the model are balanced, the length scale $\widetilde{d} \equiv C_{D E S} \Delta$ in the LES region yields a Smagorinsky eddy viscosity $\tilde{v} \propto S \Delta^{2}$. Analogous to classical LES, the role of $\Delta$. is to allow the energy cascade down to the grid size; roughly, it makes the pseudo-Kolmogorov length scale, based on the eddy viscosity, proportional to the grid spacing. The additional model constant $C_{D E S}=0.65$ was set in homogeneous turbulence ${ }^{[22]}$, and was used in the following calculations.

\section{Results}

\subsection{Abrupt Wing Stall.}

\subsubsection{Calculation Details}

As previously mentioned, the configuration examined was an $8 \%$ scale pre-production F/A-18E with $10^{\circ} / 10^{\circ} / 5^{\circ}$ flaps set. All of the calculations were carried out on a model with no vertical or horizontal stabilizer (no tails). The force coefficients presented here are compared to a no tails wind tunnel model. Wing surface pressures are compared to a wind tunnel model with tails, however there was seen to be good agreement in surface wing pressures between a model with tails, and that without. The Mach number for all cases was 0.9, and the Reynolds number was $3.8 \times 10^{6}$ per foot, leading to a chord based Reynolds number of $3.98 \times 10^{6}$. This Reynolds number was set by adjusting the freestream temperature and setting standard day sea level pressure. In order to compare frequencies and times to unsteady wind tunnel data, the resulting times in the CFD calculations were scaled by the ratio of the CFD freestream velocity to the wind tunnel freestream velocity (a factor of 1.28). The wind tunnel comparisons are from the model tested in NASA Langley's $16 \mathrm{ft}$ Transonic Tunnel (16TT). The wing was instrumented with both steady and unsteady pressure taps as shown in Figure 2. This paper will focus on the $\mathrm{G}$ row (highlighted), since it is directly behind the snag (in the streamwise direction), where the shock induced separated flow occurred furthest forward.

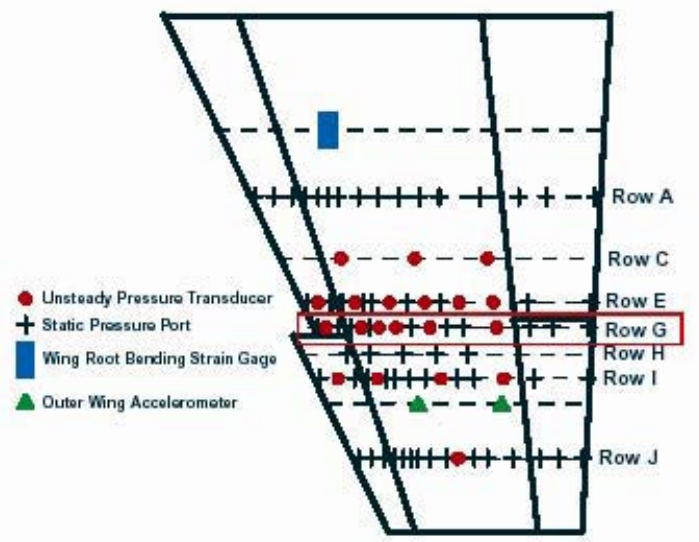

Figure 2. F/A-18E experimental pressure ports.

The grids used were unstructured grids created using the tetrahedral grid generator VGRIDns ${ }^{[23]}$. The Cobalt utility blacksmith was used to recombine the high aspect ratio tetrahedra in the boundary layer into prisms. The "Baseline" grid was $7.3 \times 10^{6}$ cells for half the aircraft. The average first $y^{+}$for the grid was 0.2 with a geometric growth rate of 1.25. An adapted grid was created in an attempt to improve on poor DES results on the baseline grid at $9^{\circ}$ angle of attack. The utility ( $\left.f v 2 u s m\right)$ was used to convert the Cobalt solution file to a format readable by Refine Mesh (a companion to VGRIDns - see Morton et $\left.a l .{ }^{[24]}\right)$. The solution used for adaption was the time averaged solution from a DES $9^{\circ}$. angle of attack run. A level of vorticity was selected that contained the separation bubble, and the grid spacing reduced by a factor of 0.6 in each coordinate direction. This should in general lead to $(1 / 0.6)^{3}=4.63$ times the number of points. However since this reduction in spacing was only applied in a narrowly focused region, the grid only increased from $7.3 \times 10^{6}$ to $9.1 \times 10^{6}$ cells. Cross sections of the "Baseline" and "Adapted" grids are shown in Figure 3. A sample instantaneous DES solution at $9^{\circ}$ angle of attack is shown in Figure 4 on the G row. The LES character of DES is clearly shown - as the grid spacing is reduced, smaller and more turbulence length scales are resolved. 
This reduces the modeling errors by increasing the resolved turbulence. By comparing Figure 3 to 4, it is also seen that the increased density of points is efficiently placed where needed - in the separation bubble. Although the adaption was carried out at a single angle of attack, the grid was used for the other angles. For lower angles, the separation bubble is further aft, so the adapted region included the separation bubble. For angles higher than $9^{\circ}$, the separation bubble was larger than the adapted region. The adaption was applied only outside the boundary layer cells.

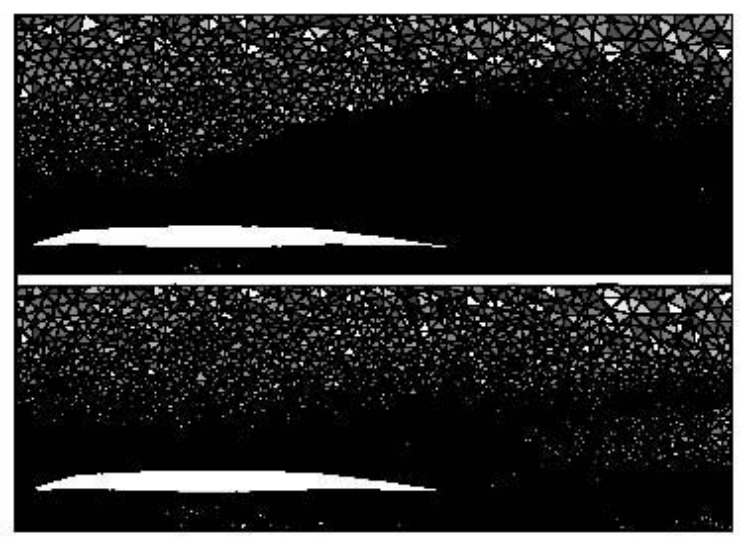

Figure 3. Baseline vs. adapted grid for F/A-18E with no tails.

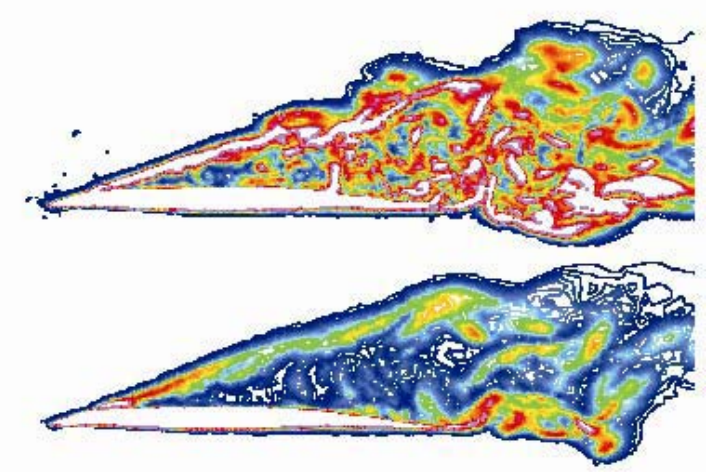

Figure 4. Vorticity contours on the baseline vs. adapted grid for F/A-18E with no tails.

For the RANS calculations, the code was run at a specified maximum global CFL of $1.0 \times 10^{6}$ to accelerate the convergence to steady state. Previous unsteady solutions using RANS models had all failed to obtain any significant levels of unsteadiness. Convergence was assessed by monitoring forces and moments during the run. When the change in forces and moments was less than $1 \%$ over 500 iterations, the solution was considered converged. This occurred between 2,000 and 4,000 iterations depending on the angle of attack.

DES calculations were of course performed timeaccurate. Three Newton subiterations were used, based on previous experience. To ensure a proper choice in timestep, a timestep study was performed on the adapted grid. The timesteps examined were $0.64 \times 10^{-5}, 1.28 \times 10^{-5}$, and $2.56 \times 10-5$ seconds. These timesteps corresponded to non-dimensional (by chord and freestream velocity) timesteps of $0.006,0.012$, and 0.024 respectively. The flow was first initialized by running the middle timestep for 4000 iterations. Then the calculations were run for 8000,4000 , and 2000 iterations respectively over the same length of physical time ( 0.0512 seconds). Power spectra of the half-aircraft rolling moment for the three timesteps is plotted in Figure 5. There is fairly poor agreement on the power at the low end of the spectra (below $100 \mathrm{~Hz}$ ) for the smallest timestep. It should be noted, however, that the length of time integrated over is quite small (only able to define $20 \mathrm{~Hz}$ ), and the low end of the spectra may need longer sampling to define it well. The middle frequency range agrees fairly well for all timesteps (between 100 and $2000 \mathrm{~Hz}$ ). The largest timestep starts to fall below the others at $2000 \mathrm{~Hz}$. This represents about 20 iterations per cycle, a reasonable value for a second order accurate code. The middle timestep falls off at about $4000 \mathrm{~Hz}$. This middle timestep is used for all the subsequent calculations. It should also be noted that this spectra provides strong evidence that DES is acting in LES mode since there is a broad range of frequencies resolved, and a healthy inertial subrange. For the subsequent DES calculations, the flow was initialized over a time of 0.0512 seconds, then time averages were taken over at least an additional 0.0512 seconds.

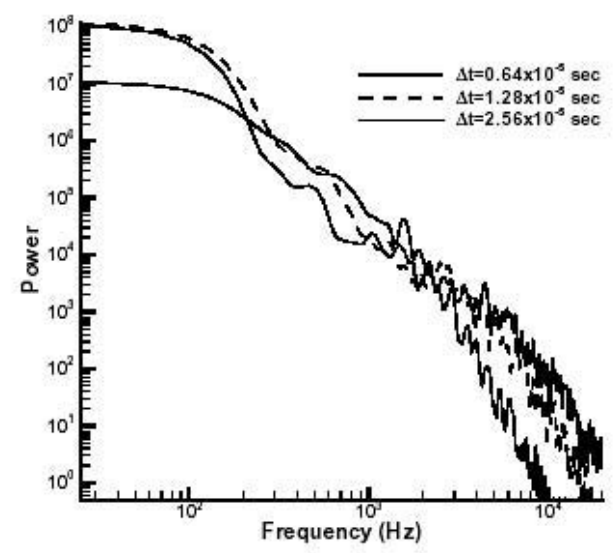

Figure 5. Power spectral density plot of half aircraft rolling moment at various timesteps, 9. angle-of-attack.

\subsubsection{Steady/Time-Averaged Results}

One of the motivating factors behind using a turbulence resolving method such as DES is to provide a more accurate time-averaged solution, mean lift and drag for example. This has proven true for a broad range of massively separated flows, such as cylinders, spheres, 
airfoils/forebodies/aircraft at high alpha, but has not been examined on a shock separated flow.

Time averaged-DES lift, drag, and moment coefficients are plotted vs. RANS calculations, and experimental values in Figures 6, 7, and 8 respectively. The experimental results were for the same configuration, i.e., without tails. The DES on the baseline grid follows the lift curve nicely up until $9^{\circ}$, where it drops in lift relative to the experiment. This discrepancy is what prompted the creation of the adapted grid, which matched the experiments better. The adapted grid matches the experiments quite well at all angles, with the largest discrepancies at $12^{\circ}$ and $16^{\circ}$ This slight error could perhaps be removed/reduced by adapting a grid to the flow solution at these angles, since the adapted grid was tailored to $9^{\circ}$, which has a smaller separation bubble than the higher angles. The Spalart- Allmaras RANS results over predict the lift at all angles, even at the low angles. Parikh and Chung ${ }^{[25]}$ performed SA calculations on an F/A-18E with the same flap settings and picks up the lift break between $9^{\circ}$ and $12^{\circ}$, where we don't have calculations. The Menter's SST model captures the low angles better but the lift curve breaks slightly early. The drag curve (Figure 7) shows essentially the same trendsover prediction by SA at all angles, an underprediction by SST near the lift break, and good agreement for the adapted DES.

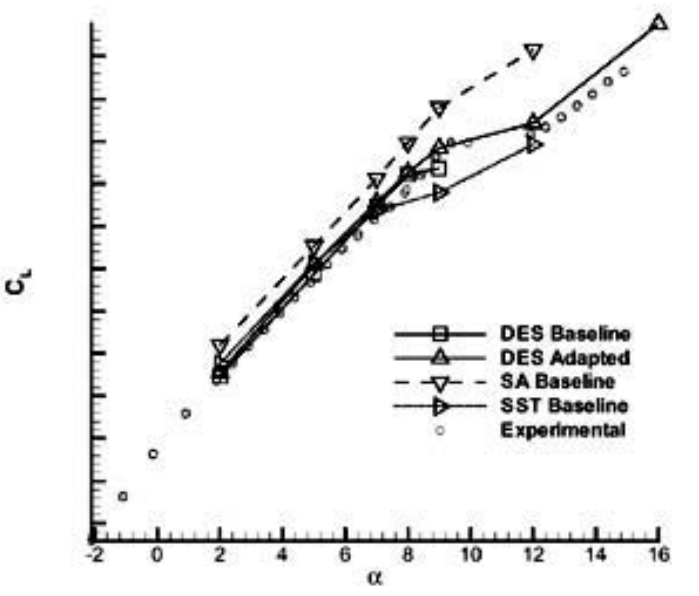

Figure 6. Lift Coefficient vs. alpha for the no tails F/A- 18E.

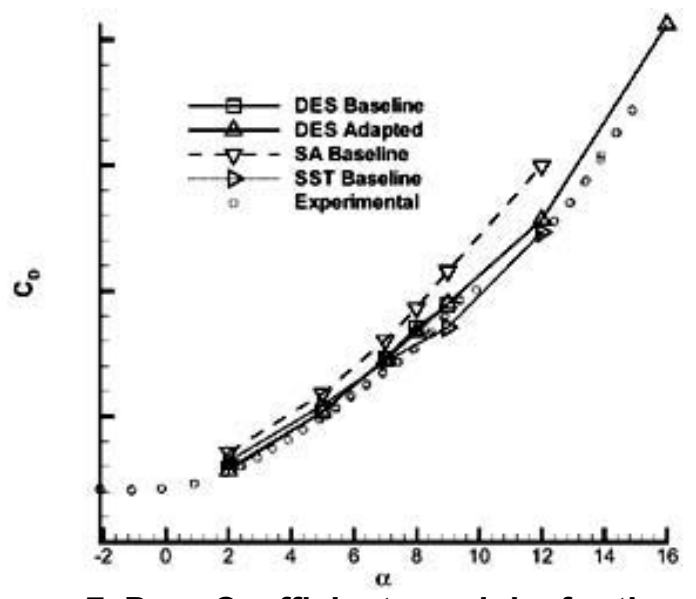

Figure 7. Drag Coefficient vs. alpha for the no tails F/A-18E.

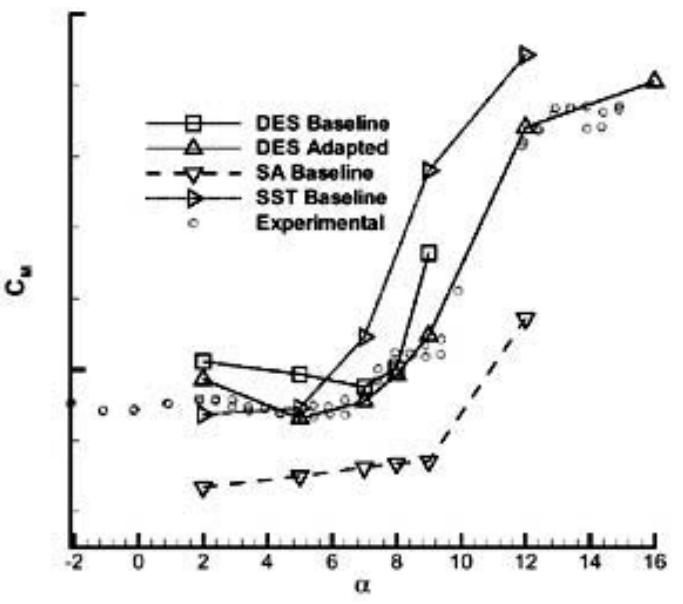

Figure 8. Pitching Moment Coefficient vs. alpha for the no tails F/A-18E.

The pitching moment coefficient (Figure 8) shows the most sensitivity to the model. Since the current grid has no tails, the moment coefficients are quite different than those presented by Parikh and Chung ${ }^{[25]}$. The adapted DES grid shows quite good agreement throughout the entire angle of attack range. SA underpredicts the moment, while SST overpredicts it at all but the two lowest angles. 


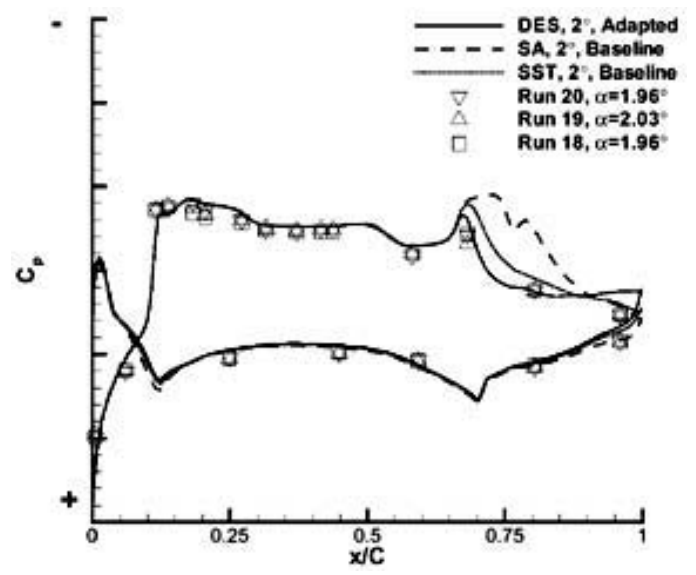

Figure 9. Time-averaged pressure coefficient vs. chord location for the no tails F/A-18E on the $G$ row, $2^{\circ}$ angle-of-attack.

To understand the differences between the models, pressure coefficients along the $\mathrm{G}$ row are plotted vs. experiments in Figures 9, 10, and 11 for $2^{\circ}, 9^{\circ}$, and $12^{\circ}$ respectively. Figure 9 suggests that experimentally there is separation over the trailing edge flap/aileron at $2^{\circ}$. Adapted DES does a good job of picking up the pressure level on the aileron correctly, although the agreement at the trailing edge is not perfect (neither is the pitching moment at this angle). SST only slightly overpredicts the pressure, hence the close but slight overprediction of lift. SA overpredicts the pressure on the flap by a significant amount, which is likely the cause for the overprediction in lift throughout the low angles.

At $9^{\circ}$ (Figure 10) the experiments show a smoothly varying pressure distribution from the snag back to about the half chord. Schuster and Byrd ${ }^{[4]}$ showed with unsteady pressure measurements that this pressure distribution occurs due to the time-averaging of an unsteady shock that moves back and forth over the wing. This is certainly a difficult effect for the RANS models to pick up. In this case both SA and SST predict relatively sharp shockswith SST separating early, and SA late. The DES adapted solution, as will be discussed in the following section, contains a moving shock, that when time-averaged gives a smeared out pressure profile. The time averaged pressures suggest that the unsteady shock stays too far forward compared to the experiments.

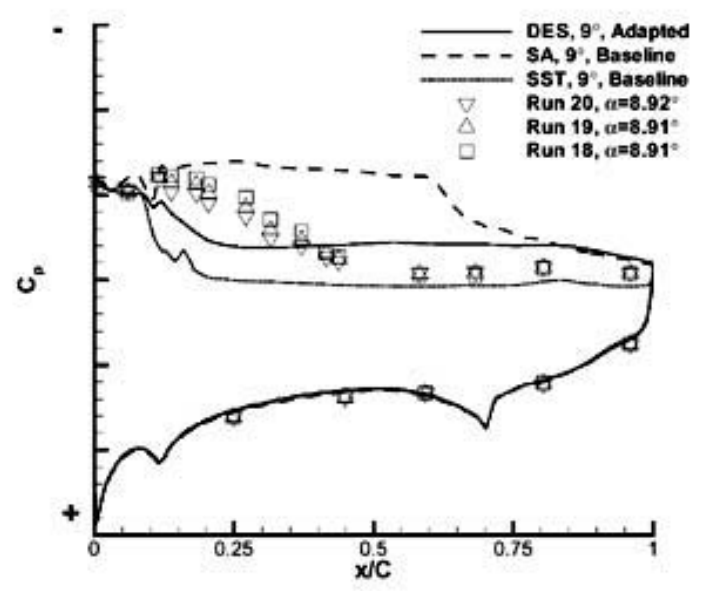

Figure 10. Time-averaged pressure coefficient vs. chord location for the no tails F/A-18E on the G row, $9^{\circ}$ angle-of-attack.

At $12^{\circ}$ (Figure 11) the flow is separated over the entire chord from the leading edge of the wing. SA overpredicts the pressure (and therefore the lift), while DES and SST match quite well. The fact that SST matches so well here suggests that the errors in pitching moment are arising from a location other than behind the region along the $\mathrm{G}$ row.

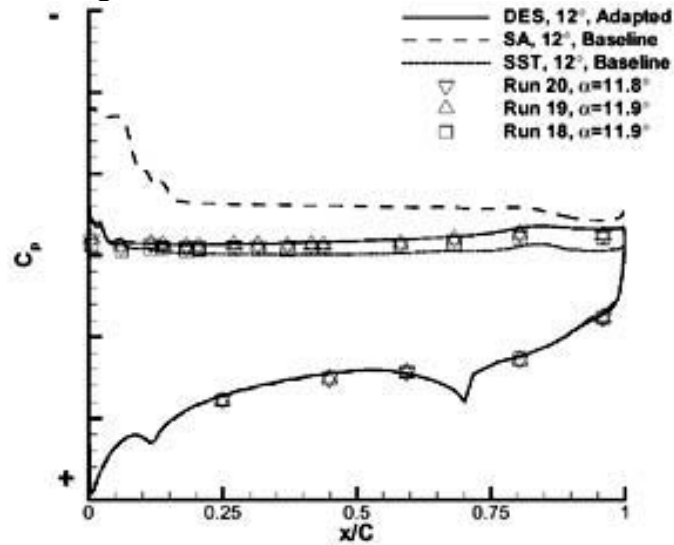

Figure 11. Time-averaged pressure coefficient vs. chord location for the no tails F/A-18E on the $\mathrm{G}$ row, $12^{\circ}$ angle-of-attack.

\subsection{Unsteady Results.}

To assess the accuracy of DES in computing unsteady effects associated with AWS, comparisons are made to the unsteady experimental data of Schuster and Byrd $^{[4]}$. The effect of the unsteady shock on the mean pressure profile is shown in Figure 12. This plot shows instantaneous pressures at four different times as well as the average pressure for the DES calculation at $9^{\circ}$ angle 
of attack. Although the instantaneous shocks are all sharp, when time averaged a smooth pressure profile results.

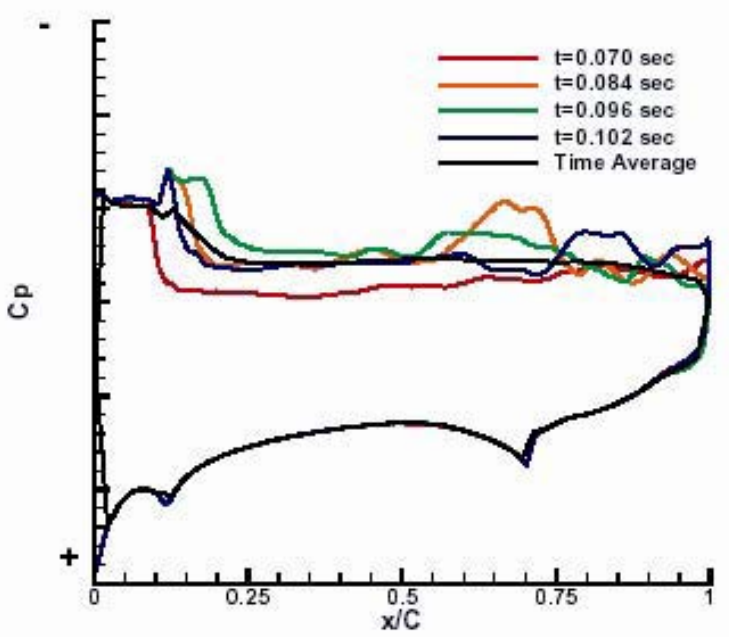

Figure 12. Pressure contours from the DES adapted calculation at four instants in time, and time-averaged at $9^{\circ}$ angle-of-attack

Comparisons between the DES calculations and the experiments are shown in Figures 13, 14, and 15. Surface pressures along the $G$ row are plotted, where the experiments had six unsteady pressure taps and ten steady taps. Additionally, there were five steady pressure taps on the bottom of the wing. It was impracticable to store the entire set of CFD results for all timesteps, so the CFD calculations were "tapped" on the G row, and pressures saved every five iterations for subsequent post processing. For the baseline calculations, only the 16 experimental taps on the top of the wing were used. For the refined grid calculations, 100 equally spaced points on the $\mathrm{G}$ row were tapped on both upper and lower surfaces to allow for more detailed analysis of the shock motion. Pressure statistics were calculated from the experiments and CFD, including the mean, standard deviation, and the minimum and maximum values of pressure. For both the CFD and experiments, any individual pressure that fell outside a three-standard-deviation $(3 \sigma)$ band about the computed mean was excluded for the maximum or minimum pressure value. For the CFD calculations this mainly smoothed out the min and max coefficients of pressure behind the shock location.

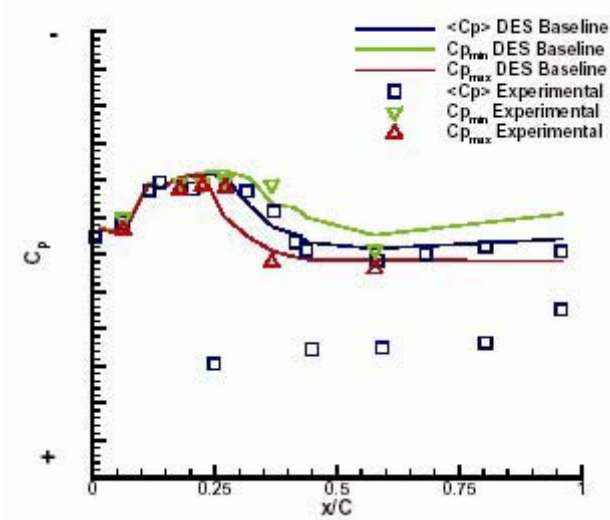

Figure 13. Min, Max, and average pressure coefficient on the $\mathrm{G}$ row, $7^{\circ}$ angle-of-attack.

Statistics at $7^{\circ}$ are plotted for the baseline grid in Figure 13. The five experimental mean pressures near the bottom of the plot are from the lower wing surface where the CFD pressures were not examined. The agreement in the mean, maximum, and minimum pressures on the top surface is quite good. The shock in the CFD is slightly too far forward and the range of pressure oscillations is slightly underpredicted.

Statistics at $9^{\circ}$ are plotted for the baseline and adapted grids in Figure 14. The oscillations in the baseline grid were underpredicted and the shock too far forward. The adapted grid helped improve the results - increasing the amount of shock oscillation, and moving the mean shock location further aft. These improvements showed up as an improved mean lift prediction as previously discussed.

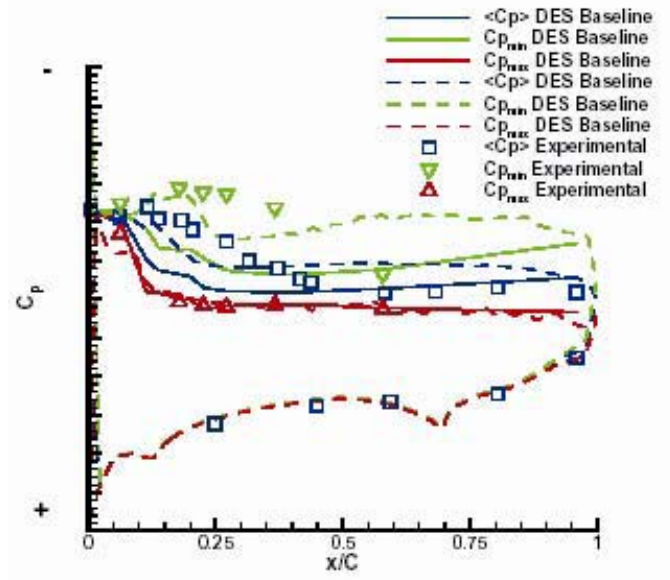

Figure 14:.Min, Max, and average pressure coefficient on the $\mathrm{G}$ row, $9^{\circ}$ angle-of-attack.

Statistics at $12^{\circ}$ are plotted for the adapted grid in Figure 15. The agreement of the maximum, minimum, 
and average pressure to the experiments is quite good. The pressures had only weak oscillation since the flow was fully separated, and there was no shock oscillation as in the $7^{\circ}$ and $9^{\circ}$ cases.

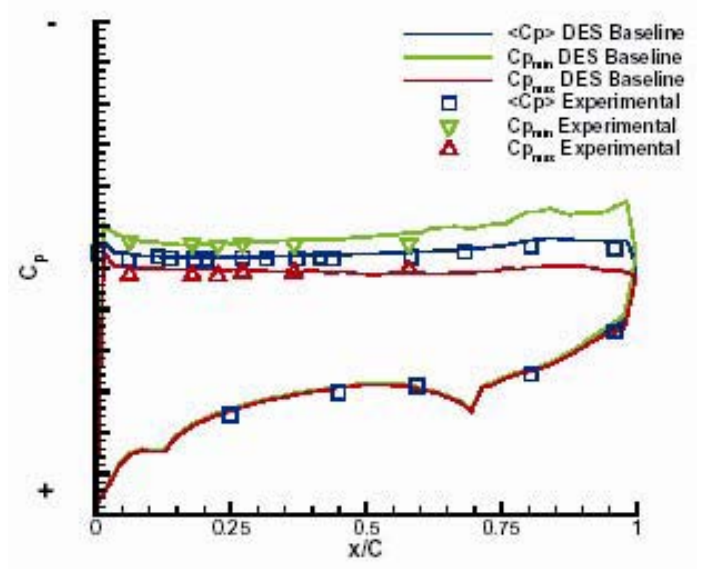

Figure 15. Min, Max, and average pressure coefficient on the $\mathrm{G}$ row, $12^{\circ}$ angle-of-attack.

To determine if unsteady shock oscillation could be a contributor to the AWS phenomenon, half-aircraft rolling moment is next examined in Figure 16. The half aircraft rolling moment was calculated by taking the rolling moment of the half-aircraft and non-dimensionalizing by the span and half the wing area. This of course leads to a non-zero mean coefficient, but a feel for the level of unsteadiness in rolling moment can be obtained by comparing the peak to peak differences. The differences in peaks in Figure 16 although not shown on the axis was considered "significantly large" and a potential contributor to triggering an AWS event. A small slice of this rolling moment plot is shown in Figure 18 with flow visualizations at seven instants in time. Figure 18a corresponds to a large rolling moment, since it has low lift, which would produce a right roll. In Figure 18b, a tiny separation bubble forms on the snag, further reducing lift and increasing the rolling moment. The shock then moves back in Figure 18c-e until the lift is at a maximum, and the rolling moment is at a minimum. From that point it moves forward in Figure 18f-g. The cycle can then repeat.

What is significant is that this shock motion causes a rolling moment change at a low frequencyapproximately $25 \mathrm{~Hz}$. This would scale to $2 \mathrm{~Hz}$ for the full scale aircraft. This was however only a half aircraft calculation, so care must be taken in drawing conclusions from this plot. The net rolling moment will depend on the flow on 